\title{
IRREDUCIBLE SEMIGROUPS OF FUNCTIONALLY POSITIVE NILPOTENT OPERATORS
}

\author{
YONG ZHONG
}

\begin{abstract}
For each irrational number $\theta \in(0,1)$, we construct a semigroup $\mathscr{S}_{\theta}$ of nilpotent operators on $\mathscr{L}^{2}([0,1])$ that are also partial isometries and positive in the sense that the operator maps nonnegative functions to nonnegative functions. We prove that each semigroup $\mathscr{S}_{\theta}$ is discrete in the norm topology and hence norm-closed and that the weak closure of $\mathscr{S}_{\theta}$ is independent of $\theta$. We show that each semigroup $\mathscr{S}_{\theta}$ has no nontrivial invariant subspaces.
\end{abstract}

Consider the Hilbert space $\mathscr{L}^{2}([0,1])$ with the Lebesgue measure $m$ on $[0,1]$. An operator $T$ on $\mathscr{L}^{2}([0,1])$ is called functionally positive (or simply, positive) if $T$ maps nonnegative functions to nonnegative functions. It has been proven in [5] that certain multiplicative semigroups of positive quasinilpotent operators are reducible, that is, all operators in the semigroup have a common nontrivial invariant subspace. One may ask: Is every semigroup of positive quasinilpotent operators reducible?

In [1, Theorem 1], Hadwin et al. constructed an irreducible semigroup of nilpotent operators on a Hilbert space such that every operator in the semigroup has nilpotency two but is not positive in any sense. And in [3], Schaefer provided a method of constructing a positive quasinilpotent operator on the Hilbert space of $L^{2}$-functions of the unit circle such that the operator does not have any nontrivial invariant subspaces corresponding to projections that are multiplication operators induced by characteristic functions on the unit circle. It is easy to see that neither of the two examples answers the above question. In this paper, we answer the question negatively by constructing an irreducible semigroup of positive nilpotent operators that are also partial isometries.

For every $\alpha \in[0,1]$, we define $S_{\alpha}$ and $T_{\alpha}$ as follows:

$$
\begin{gathered}
\left(S_{\alpha} f\right)(t)=\left\{\begin{array}{ll}
f(t+\alpha) & \text { if } t \in[0,1-\alpha], \\
0 & \text { if } t \in(1-\alpha, 1],
\end{array} \quad f \in \mathscr{L}^{2}([0,1]),\right. \\
\left(T_{\alpha} f\right)(t)=\left\{\begin{array}{ll}
0 & \text { if } t \in[0, \alpha), \\
f(t-\alpha) & \text { if } t \in[\alpha, 1],
\end{array} \quad f \in \mathscr{L}^{2}([0,1]) .\right.
\end{gathered}
$$

Received by the editors December 13,1993; originally communicated to the Proceedings of the $A M S$ by Palle E. T. Jorgensen.

1991 Mathematics Subject Classification. Primary 47A15; Secondary 47D03.

Key words and phrases. Invariant subspace, nilpotent operator, semigroup of operators.

The results of this paper constitute a portion of the author's Ph.D. dissertation written under the supervision of Heydar Radjavi, Dalhousie University. 
Clearly, $S_{\alpha}$ and $T_{\alpha}$ are well-defined bounded linear operators on $\mathscr{L}^{2}([0,1])$. For convenience, we define $S_{\alpha}=T_{\alpha}=0$ for all $\alpha>1$. Still, we denote by $M_{\phi}$ the multiplication operator corresponding to $\phi \in \mathscr{L}^{\infty}([0,1], m)$.

Lemma 1. For any $\alpha \in[0,1]$,

(i) $S_{\alpha}$ and $T_{\alpha}$ are positive operators.

(ii) $S_{\alpha}^{*}=T_{\alpha}, S_{0}=T_{0}=I, S_{1}=T_{1}=0$.

(iii) $S_{\alpha} T_{\alpha}=M_{\chi_{[0,1-\alpha]}}, T_{\alpha} S_{\alpha}=M_{\chi_{[\alpha, 1]}}$, and therefore, $S_{\alpha}$ and $T_{\alpha}$ are partial isometries.

(iv) If $\alpha \neq 1$, then $\left\|S_{\alpha}\right\|=\left\|T_{\alpha}\right\|=1$.

Proof. (i) It is obvious that $S_{\alpha}$ and $T_{\alpha}$ are positive operators.

(ii) For any $f, g \in \mathscr{L}^{2}([0,1])$,

$$
\begin{aligned}
\left\langle S_{\alpha} f, g\right\rangle & =\int_{0}^{1}\left(S_{\alpha} f\right)(t) \bar{g}(t) d t \\
& =\int_{0}^{1-\alpha} f(t+\alpha) \bar{g}(t) d t \\
& =\int_{\alpha}^{1} f(s) \bar{g}(s-\alpha) d s \\
& =\left\langle f, T_{\alpha} g\right\rangle .
\end{aligned}
$$

Thus, $S_{\alpha}^{*}=T_{\alpha}$. Clearly, $S_{0}=T_{0}=I, S_{1}=T_{1}=0$.

(iii) For any $f, g \in \mathscr{L}^{2}([0,1])$,

$$
\begin{aligned}
\left\langle S_{\alpha} T_{\alpha} f, g\right\rangle & =\left\langle T_{\alpha} f, T_{\alpha} g\right\rangle \\
& =\int_{\alpha}^{1} f(t-\alpha) \bar{g}(t-\alpha) d t \\
& =\int_{0}^{1-\alpha} f(s) \bar{g}(s) d s \\
& =\left\langle M_{\chi_{[0,1-\alpha]}} f, g\right\rangle .
\end{aligned}
$$

Therefore, $S_{\alpha} T_{\alpha}=M_{\chi_{[0,1-\alpha]}}$. Similarly, $T_{\alpha} S_{\alpha}=M_{\chi_{[a, 1]}}$.

Since

$$
S_{\alpha}^{*} S_{\alpha}=T_{\alpha} S_{\alpha}=M_{\chi_{[\alpha, 1]}}, \quad T_{\alpha}^{*} T_{\alpha}=S_{\alpha} T_{\alpha}=M_{\chi_{[0,1-\alpha]}}
$$

are projections, we have that $S_{\alpha}$ and $T_{\alpha}$ are partial isometries.

(iv) It follows immediately from (iii).

Lemma 2. For any $\alpha \in[0,1)$ and any $\phi \in \mathscr{L}^{\infty}([0,1])$,

(i) $S_{\alpha} M_{\phi}=M_{S_{\alpha} \phi} S_{\alpha}, T_{\alpha} M_{\phi}=M_{T_{\alpha} \phi} T_{\alpha}$.

(ii) $M_{\phi} S_{\alpha}, S_{\alpha} M_{\phi}, M_{\phi} T_{\alpha}$, and $T_{\alpha} M_{\phi}$ are all nilpotent operators.

Proof. (i) For any $f \in \mathscr{L}^{2}([0,1])$,

$$
S_{\alpha}(\phi f)=\left(S_{\alpha} \phi\right)\left(S_{\alpha} f\right) .
$$

Therefore,

$$
\left(S_{\alpha} M_{\phi}\right) f=S_{\alpha}(\phi f)=\left(S_{\alpha} \phi\right)\left(S_{\alpha} f\right)=\left(M_{S_{\alpha} \phi} S_{\alpha}\right) f .
$$

Hence, $S_{\alpha} M_{\phi}=M_{S_{\alpha} \phi} S_{\alpha}$. Similarly, $T_{\alpha} M_{\phi}=M_{T_{\alpha} \phi} T_{\alpha}$. 
(ii) It is obvious that $\left(S_{\alpha}\right)^{p}=S_{p \alpha}$ for any positive integer $p$. Therefore, it follows from (i) that

$$
\left(M_{\phi} S_{\alpha}\right)^{p}=M_{\phi} M_{S_{\alpha} \phi} \cdots M_{S_{(p-1) \alpha} \phi} S_{p \alpha}
$$

for any positive integer $p$. Hence, $\left(M_{\phi} S_{\alpha}\right)^{p}=0$ for $p$ large enough to satisfy $p \alpha>1$. Thus, $M_{\phi} S_{\alpha}$ is a nilpotent operator. Similarly, $S_{\alpha} M_{\phi}, M_{\phi} T_{\alpha}$, and $T_{\alpha} M_{\phi}$ are all nilpotent operators.

Lemma 3. If $\alpha, \beta \in[0,1]$, then

(i) $S_{\alpha} S_{\beta}=S_{\alpha+\beta}$ and $T_{\alpha} T_{\beta}=T_{\alpha+\beta}$.

(ii)

(iii)

$$
S_{\alpha} T_{\beta}= \begin{cases}M_{\chi_{[0,1-\alpha]}} T_{\beta-\alpha} & \text { if } \alpha \leq \beta, \\ M_{\chi_{[0,1-\alpha]}} S_{\alpha-\beta} & \text { if } \alpha>\beta .\end{cases}
$$

$$
T_{\beta} S_{\alpha}= \begin{cases}M_{\chi_{[\beta, 1]}} T_{\beta-\alpha} & \text { if } \alpha \leq \beta \\ M_{\chi_{[\beta, 1]}} S_{\alpha-\beta} & \text { if } \alpha>\beta\end{cases}
$$

Proof. (i) It is easy to check.

(ii) If $\alpha \leq \beta$, then by (i) and Lemma 1

$$
S_{\alpha} T_{\beta}=S_{\alpha} T_{\alpha} T_{\beta-\alpha}=M_{\chi_{[0,1-\alpha]}} T_{\beta-\alpha} .
$$

If $\alpha>\beta$, then by (i), Lemma 1 and Lemma 2 ,

$$
\begin{aligned}
S_{\alpha} T_{\beta} & =S_{\alpha-\beta} S_{\beta} T_{\beta}=S_{\alpha-\beta} M_{\chi_{[0,1-\beta]}} \\
& =M_{S_{\alpha-\beta} \chi_{[0,1-\beta]}} S_{\alpha-\beta}=M_{\chi_{[0,1-\alpha]}} S_{\alpha-\beta} .
\end{aligned}
$$

(iii) The proof is similar to that of (ii).

In [4] it was proved that every positive operator $S$ on $\mathscr{L}^{2}([0,1])$ is a pseudo-integral operator and that $S$ is determined by a positive finite Borel measure $\mu_{S}$ on $[0,1] \times[0,1]$ by the equation

$$
\langle S f, g\rangle=\int_{[0,1] \times[0,1]} f(y) \bar{g}(x) \mu_{S}(d x, d y) .
$$

For any $\alpha \in[0,1]$, let

$$
G_{\alpha}=\{(x, y) \in[0,1] \times[0,1]: y=x+\alpha\}
$$

and

$$
F_{\alpha}=\{(x, y) \in[0,1] \times[0,1]: y=x-\alpha\} .
$$

It is easy to check that $S_{\alpha}$ is a pseudo-integral operator determined by $\mu_{\alpha}$, where $\mu_{\alpha}$ is the positive finite Borel measure defined by the equation

$$
\mu_{\alpha}(E)=m\left(\left\{x \in[0,1]:(x, y) \in E \cap G_{\alpha} \text { for some } y \in[0,1]\right\}\right) .
$$

Similarly, $T_{\alpha}$ is a pseudo-integral operator determined by $\nu_{\alpha}$, where $\nu_{\alpha}$ is defined by the equation

$$
\nu_{\alpha}(E)=m\left(\left\{x \in[0,1]:(x, y) \in E \cap F_{\alpha} \text { for some } y \in[0,1]\right\}\right) .
$$

Next we construct a multiplicative semigroup of positive nilpotent operators that are also partial isometries and prove that the semigroup is irreducible. 
Choose an arbitrary irrational number $\theta \in(0,1)$. Let $\mathscr{S}_{\theta}$ be the multiplicative semigroup generated by the set

$$
\left\{S_{a}, T_{b \theta}: a, b \in(0,1) \text { are rational numbers }\right\} .
$$

Theorem 4. Suppose $\theta \in(0,1)$ is irrational. Then the semigroup $\mathscr{S}_{\theta}$ consists of positive nilpotent operators that are also partial isometries.

Proof. Easy to see that every operator in $\mathscr{S}_{\theta}$ is a product of positive operators and, therefore, positive itself.

By Lemma 3 (i), any 'word' in $\mathscr{S}_{\theta}$ looks like

$$
W=S_{a_{1}}^{p_{1}} T_{b_{1} \theta}^{q_{1}} S_{a_{2}}^{p_{2}} T_{b_{2} \theta}^{q_{2}} \cdots S_{a_{n}}^{p_{n}} T_{b_{n} \theta}^{q_{n}}
$$

for some integer $n \geq 1$, where $a_{j}, b_{j} \in(0,1)$ are rational numbers and $p_{j}$, $q_{j}$ are nonnegative integers for all $j=1,2, \ldots, n$ and at least one of $p_{j}, q_{j}$ is nonzero $(j=1,2, \ldots, n)$.

By Lemma 3, $W$ is either 0 , or $M_{\phi} S_{a-b}$ with $a-b>0$, or $M_{\psi} T_{b-a}$ with $a-b<0$, where $a=\sum_{j=1}^{n} p_{j} a_{j}, b=\theta \sum_{j=1}^{n} q_{j} b_{j}$, and $\phi$ and $\psi$ are characteristic functions of some intervals. Hence $W$ is a partial isometry. Clearly, $a \neq b$ since $\theta$ is irrational, and thus, by Lemma 2 (ii), $W$ is a nilpotent operator.

Now we prove that $\mathscr{S}_{\theta}$ is discrete and irreducible. To do this, we need the following lemmas.

Lemma 5. Let $\alpha \in[0,1]$ and $[a, b]$ be an interval in $[0,1]$. Then $\left\|M_{\chi_{[a, b]}} S_{\alpha}\right\|$ $=1$ if $M_{\chi_{[a, b]}} S_{\alpha} \neq 0$, and $\left\|M_{\chi_{[a, b]}} T_{\alpha}\right\|=1$ if $M_{\chi_{[a, b]}} T_{\alpha} \neq 0$.

Proof. We only provide here the proof of the first part of this lemma. Since the range of $S_{\alpha}$ is $\chi_{[0,1-\alpha]} \mathscr{L}^{2}([0,1])$, the interval $\left[a^{\prime}, b^{\prime}\right]=[a, b] \cap[0,1-\alpha]$ has length $b^{\prime}-a^{\prime}>0$ if $M_{\chi[a, b]} S_{\alpha} \neq 0$ and

$$
M_{\chi_{\left[a^{\prime}, b^{\prime}\right]}} S_{\alpha}=M_{\chi_{[a, b]}} S_{\alpha} .
$$

Clearly, $\left\|M_{\chi_{[a, b]}} S_{\alpha \alpha}\right\| \leq\left\|M_{\chi_{[a, b]}}\right\|\left\|S_{\alpha \alpha}\right\|=1$. fore,

Let $f=\chi_{\left[a^{\prime}+\alpha, b^{\prime}+\alpha\right]}$. Then $\|f\|=\left\|\chi_{\left[a^{\prime}, b^{\prime}\right]}\right\| \neq 0$ and $S_{\alpha x} f=\chi_{\left[a^{\prime}, b^{\prime}\right]}$. There-

$$
\left\|\left(M_{\chi_{[a, b]}} S_{\alpha \alpha}\right) f\right\|=\left\|\left(M_{\chi_{\left[a^{\prime}, b^{\prime}\right]}} S_{\alpha}\right) f\right\|=\left\|M_{\chi_{\left[a^{\prime}, h^{\prime}\right]}} \chi_{\left[a^{\prime}, b^{\prime}\right]}\right\|=\|f\|,
$$

and hence, $\left\|M_{\chi_{[a, b]}} S_{c \mid}\right\| \geq 1$. It follows that $\left\|M_{\chi_{[a, b]}} S_{c}\right\|=1$.

Lemma 6. Suppose $\alpha, \beta \in[0,1]$ and $E, F$ are two intervals in $[0,1]$ such that $M_{\chi_{E}} S_{\alpha} \neq M_{\chi_{F}} T_{\beta}$. Then $\left\|M_{\chi_{F}} S_{\alpha}-M_{\chi_{F}} T_{\beta}\right\|=1$.

Proof. If either $M_{\chi_{k}} S_{\alpha}$ or $M_{\chi_{F}} T_{\beta}$ is 0 , then we are done by Lemma 5.

Suppose $M_{\chi_{E}} S_{\alpha} \neq 0$ and $M_{\chi_{k}} T_{\beta} \neq 0$. Then both $E^{\prime}=E \cap[0,1-\alpha]$ and $F^{\prime}=F \cap[\beta, 1]$ are intervals of length greater than 0 . If $\alpha=\beta=0$, then $S_{\alpha}=T_{\beta}=I$, and therefore, the result is obviously true. Thus we may assume that $\alpha+\beta>0$. By the definition of $E^{\prime}$, we can choose an interval $[a, b]$ satisfying $0<b-a<\alpha+\beta$ and

$$
[a, b] \subseteq E^{\prime}+\alpha \subseteq[\alpha, 1] .
$$

Hence

$$
[a, b]-\alpha=[a-\alpha, b-\alpha] \subseteq E^{\prime}
$$


and, because $b-\alpha<a+\beta$,

$$
([a, b]-\alpha) \cap([a, b]+\beta)=[a-\alpha, b-\alpha] \cap[a+\beta, b+\beta]=\varnothing .
$$

Let $f=\chi_{[a, b]}$. Then $f \neq 0$ and

$$
\begin{aligned}
\left\|\left(M_{\chi_{E}} S_{\alpha}-M_{\chi_{F}} T_{\beta}\right) f\right\|^{2} & =\left\|\left(M_{\chi_{E^{\prime}}} S_{\alpha}-M_{\chi_{F^{\prime}}} T_{\beta}\right) f\right\|^{2} \\
& =\left\|\chi_{E^{\prime}} \chi_{[a, b]-\alpha}-\chi_{F^{\prime}} \chi_{[a, b]+\beta}\right\|^{2} \\
& =\left\|\chi_{[a, b]-\alpha}-\chi_{F^{\prime} \cap([a, b]+\beta)}\right\|^{2} \\
& =\left\|\chi_{[a, b]-\alpha}\right\|^{2}+\left\|\chi_{F^{\prime} \cap([a, b]+\beta)}\right\|^{2} \\
& \geq\left\|\chi_{[a, b]-\alpha}\right\|^{2} \\
& =\left\|\chi_{[a, b]}\right\|^{2} \\
& =\|f\|^{2} .
\end{aligned}
$$

It follows that $\left\|M_{\chi_{E}} S_{\alpha}-M_{\chi_{F}} T_{\beta}\right\| \geq 1$.

Lemma 7. Suppose $\alpha, \beta \in[0,1]$ and $E, F$ are two intervals in $[0,1]$. Then $\left\|M_{\chi E} S_{\alpha}-M_{\chi_{F}} S_{\beta}\right\| \geq 1$ if $M_{\chi_{E}} S_{\alpha} \neq M_{\chi_{F}} S_{\beta}$ and $\left\|M_{\chi_{E}} T_{\alpha}-M_{\chi_{F}} T_{\beta}\right\| \geq 1$ if $M_{\chi E} T_{\alpha} \neq M_{\chi F} T_{\beta}$.

Proof. For the first part of the lemma, if either $\alpha$ or $\beta$ is 0 or 1 , then we are done by Lemma 5, Lemma 6 and the fact that $S_{0}=T_{0}=I$. So we may assume that $0<\alpha \leq \beta<1$. Therefore, by (ii) of Lemma 3,

$$
\begin{aligned}
\left\|M_{\chi_{E}} S_{\alpha}-M_{\chi_{F}} S_{\beta}\right\| & =\left\|M_{\chi_{E}} S_{\alpha}-M_{\chi_{F}} S_{\beta}\right\|\left\|T_{\alpha}\right\| \\
& \geq\left\|M_{\chi_{E}} S_{\alpha} T_{\alpha}-M_{\chi_{F}} S_{\beta} T_{\alpha}\right\| \\
& =\left\|M_{\chi_{E}} M_{\chi_{[0,1-\alpha]}}-M_{\chi_{F}} M_{\chi_{[0,1-\beta]}} S_{\beta-\alpha}\right\| \\
& =\left\|M_{\left.\chi_{E} \cap 0,1-\alpha\right]} T_{0}-M_{\chi_{F \cap[0,1-\beta]}} S_{\beta-\alpha}\right\| .
\end{aligned}
$$

By Lemma 6, either

$$
\left\|M_{\chi E \cap[0,1-\alpha]} T_{0}-M_{\chi F \cap[0,1-\beta]} S_{\beta-\alpha}\right\| \geq 1
$$

or

$$
M_{\chi E \cap[0,1-a]} T_{0}-M_{\chi F \cap[0,1-\beta]} S_{\beta-\alpha}=0 .
$$

Thus, either

$$
\left\|M_{\chi E} S_{\alpha}-M_{\chi F} S_{\beta}\right\| \geq 1
$$

or

$$
\begin{aligned}
M_{\chi E} S_{\alpha}-M_{\chi_{F}} S_{\beta} & =M_{\chi E \cap[0,1-\alpha]} S_{\alpha}-M_{\chi_{F \cap[0.1-\beta]}} S_{\beta} \\
& =\left(M_{\chi E \cap[0,1-a]}-M_{\chi_{F \cap[0,1-\beta]}} S_{\beta-\alpha}\right) S_{\alpha} \\
& =0 .
\end{aligned}
$$

Since $T_{\alpha}=\left(S_{\alpha}\right)^{*}$ and $T_{\beta}=\left(S_{\beta}\right)^{*}$, we have

$$
\begin{aligned}
\left(M_{\chi_{E}} T_{\alpha}-M_{\chi_{F}} T_{\beta}\right)^{*} & =S_{\alpha} M_{\chi_{k}}-S_{\beta} M_{\chi_{F}} \\
& =M_{S_{u} \chi_{E}} S_{\alpha}-M_{S_{\beta} \chi_{F}} S_{\beta} \\
& =M_{\chi_{E-\alpha}} S_{\alpha}-M_{\chi_{F-\beta}} S_{\beta} .
\end{aligned}
$$

Thus, the second part of the lemma follows directly from the first part. 
Theorem 8. Suppose $\theta \in(0,1)$ is irrational. Then the norm-distance between any two distinct elements of $\mathscr{S}_{\theta}$ is at least 1 . Therefore, the semigroup $\mathscr{S}_{\theta}$ is discrete and, hence, norm-closed in $\mathscr{B}\left(\mathscr{L}^{2}([0,1])\right)$.

Proof. From the proof of Theorem 4, any element in $\mathscr{S}_{\theta}$ is of the form $M_{\chi_{E}} S_{\alpha}$ or $M_{\chi_{F}} T_{\beta}$ where $E$ and $F$ are intervals in $[0,1]$ and $\alpha=a-b \theta, \beta=c \theta-d$ are in $[0,1]$ for some rational numbers $a, b, c$, and $d$. Thus, the result follows immediately from Lemma 6 and Lemma 7.

Theorem 9. Suppose $\theta \in(0,1)$ is irrational. Then for any $\alpha \in[0,1], S_{\alpha}$ and $T_{\alpha}$ are in the weak closure $\overline{\mathscr{S}}_{\theta}^{\text {WOT }}$ of $\mathscr{S}_{\theta}$. Consequently, $\overline{\mathscr{S}}_{\theta}{ }^{\mathrm{WOT}}$ is selfadjoint and independent of $\theta$.

Proof. Clearly, $S_{1}=T_{1}=0 \in \mathscr{S}_{\theta}$.

For any $\alpha \in[0,1)$, choose a decreasing sequence $\left\{a_{j}\right\}$ of rational numbers in $(0,1)$ such that $\lim a_{j}=\alpha$. We claim that $S_{\alpha}$ is the weak limit of the sequence $\left\{S_{a_{j}}\right\}$, and hence, $S_{\alpha} \in \overline{\mathscr{S}}_{\boldsymbol{\theta}}^{\text {WOT }}$.

We need to show that

$$
\left\langle S_{a_{j}} f, g\right\rangle \rightarrow\left\langle S_{\alpha} f, g\right\rangle \quad(j \rightarrow \infty)
$$

for all $f$ and $g$ in $\mathscr{L}^{2}([0,1])$. Since $\left\|S_{a_{j}}\right\|=1$ for all $j$ and since $C([0,1])$ is dense in $\mathscr{L}^{2}([0,1])$, it suffices to show that

$$
\left\langle S_{a_{j}} f, g\right\rangle \rightarrow\left\langle S_{\alpha} f, g\right\rangle \quad(j \rightarrow \infty)
$$

for all $f$ and $g$ in $C([0,1])$.

Suppose $f$ and $g$ are in $C([0,1])$. For any positive number $\varepsilon>0$, by the continuity of $f$ we can find a number $\delta>0$ such that

$$
|f(x)-f(y)|<\varepsilon
$$

whenever $x, y \in[0,1]$ and $|x-y|<\delta$. Since $\lim a_{j}=\alpha$, we can find a positive integer $N$ such that

$$
\left|a_{j}-\alpha\right|<\min (\varepsilon, \delta)
$$

for all $j$ with $j \geq N$.

Therefore, for any $j, j \geq N$,

$$
\begin{aligned}
\mid\left\langle S_{a_{j}} f\right. & , g\rangle-\left\langle S_{\alpha} f, g\right\rangle \mid \\
= & \left|\int_{0}^{1-a_{j}} f\left(x+a_{j}\right) \bar{g}(x) d x-\int_{0}^{1-\alpha} f(x+\alpha) \bar{g}(x) d x\right| \\
= & \mid \int_{0}^{1-a_{j}} f\left(x+a_{j}\right) \bar{g}(x) d x-\int_{0}^{1-a_{j}} f(x+\alpha) \bar{g}(x) d x \\
& \quad+\int_{1-a_{j}}^{1-\alpha} f(x+\alpha) \bar{g}(x) d x \mid \\
= & \int_{0}^{1-a_{j}}\left|f\left(x+a_{j}\right)-f(x+\alpha)\right||\bar{g}(x)| d x \\
& +\int_{1-a_{j}}^{1-\alpha}|f(x+\alpha)||\bar{g}(x)| d x \\
\leq & \varepsilon\|g\|_{\infty}+\left(a_{j}-\alpha\right)\|f\|_{\infty}\|g\|_{\infty} \\
\leq & \varepsilon\|g\|_{\infty}\left(1+\|f\|_{\infty}\right) .
\end{aligned}
$$


Thus,

$$
\left\langle S_{a_{j}} f, g\right\rangle \rightarrow\left\langle S_{\alpha} f, g\right\rangle \quad(j \rightarrow \infty) .
$$

Similarly, by choosing a decreasing sequence $\left\{b_{j}\right\}$ of rational numbers in $(0,1)$ with $\lim b_{j} \theta=\alpha$, we can prove that $T_{\alpha} \in \overline{\mathscr{S}}_{\theta}^{\text {WOT }}$.

Since $S_{\alpha}$ and $T_{\alpha}$ are in $\overline{\mathscr{S}}_{\theta}^{\text {WOT }}$ and $S_{\alpha}^{*}=T_{\alpha}$ for any $\alpha \in[0,1]$, we have that $\overline{\mathscr{S}}_{\theta}$ WOT is selfadjoint.

We now prove that $\overline{\mathscr{S}}_{\theta}^{\text {WOT }}$ is independent of $\theta$. Let $\theta_{1}$ and $\theta_{2}$ be two irrational numbers in $(0,1)$. For every $\alpha \in[0,1]$, by what we just proved, $S_{\alpha}$ and $T_{\alpha}$ are the weak limits of sequences of operators in $\mathscr{S}_{\theta_{1}}$. Let $W$ be an arbitrary operator in $\mathscr{S}_{\theta_{2}}$. To prove that $W$ is in $\overline{\mathscr{S}}_{\theta_{1}}{ }^{\text {WOT }}$, we may assume that $W \neq 0$. From the proof of Theorem $4, W$ is in the form of $M_{\chi_{[a, b]}} S_{\alpha}$ or $M_{\chi_{[a, b]}} T_{\alpha}$ for some interval $[a, b] \subseteq[0,1]$ and some number $\alpha \in[0,1)$. Choose a sequence $\left\{\left[a_{j}, b_{j}\right]\right\}$ of subintervals of $[a, b]$ with the property that $\lim a_{j}=a$ and $\lim b_{j}=b$. Then it is easy to check that $M_{\chi_{[a, b]}}$ is the strong

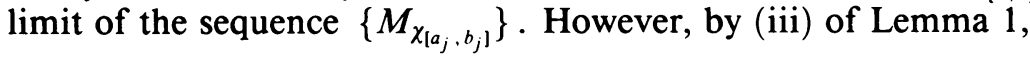

$$
M_{\chi_{\left[a_{j}, b_{j}\right]}}=M_{\chi_{\left[a_{j}, 1\right]}} M_{\chi_{\left[0, b_{j}\right]}}=T_{a_{j}} S_{a_{j}} S_{1-b_{j}} T_{1-b_{j}}
$$

for every integer $j$. We can choose $\left\{\left[a_{j}, b_{j}\right]\right\}$ so that all $M_{\chi_{\left[a_{j}, b_{j}\right]}}$ are in $\mathscr{S}_{\theta_{1}}$ because $\mathscr{S}_{\theta_{1}}$ is a semigroup. Thus $M_{\chi_{[a, b]}}$ is the strong limit of a sequence of operators in $\mathscr{S}_{\theta_{1}}$. It follows that $W$ is the weak limit of a sequence of operators in $\mathscr{S}_{\theta_{1}}$, and hence $\mathscr{S}_{\theta_{2}} \subseteq \overline{\mathscr{S}}_{\theta_{1}}^{\text {WOT }}$. Consequently, $\overline{\mathscr{S}}_{\theta_{2}}^{\text {WOT }} \subseteq \overline{\mathscr{S}}_{\theta_{1}}^{\text {WOT }}$.

Similarly, we have $\overline{\mathscr{S}}_{\theta_{1}}^{\text {WOT }} \subseteq{\overline{\mathscr{S}_{\theta_{2}}}}^{\text {WOT }}$. Therefore, $\overline{\mathscr{S}}_{\theta_{1}}^{\text {WOT }}={\overline{\mathscr{S}_{\theta_{2}}}}^{\text {WOT }}$.

Theorem 10. Suppose $\theta \in(0,1)$ is irrational. Then the algebra generated by the semigroup $\mathscr{S}_{\theta}$ is weakly dense in $\mathscr{B}\left(\mathscr{L}^{2}([0,1])\right)$.

Proof. Let $\mathscr{A}$ be the weakly closed algebra generated by the semigroup $\mathscr{S}_{\theta}$. Then $\overline{\mathscr{S}}_{\theta}^{\text {WOT }} \subseteq \mathscr{A}$. It follows that $\mathscr{A}$ is selfadjoint. To prove $\mathscr{A}=$ $\mathscr{B}\left(\mathscr{L}^{2}([0,1])\right)$, we only need to show that the commutant $\mathscr{A}^{\prime}$ of $\mathscr{A}$ is trivial.

Since $\overline{\mathscr{S}}_{\theta}{ }^{\text {WOT }} \subseteq \mathscr{A}$ and $S_{\alpha}$ and $T_{\alpha}$ are in $\overline{\mathscr{S}}_{\theta}^{\text {WOT }}$ for any $\alpha \in[0,1]$, we have that $\mathscr{A}$ contains all multiplication operators corresponding to characteristic functions of intervals in $[0,1]$ by (iii) of Lemma 1 . Thus $\mathscr{A}$ contains all multiplication operators $M_{\phi}$ with $\phi \in \mathscr{L}^{\infty}([0,1])$. It follows that any projection in the commutant $\mathscr{A}^{\prime}$ is of the form $M_{\chi_{E}}$ for some measurable set $E \subseteq[0,1]$.

Let $E$ be a measurable set in $[0,1]$ such that $M_{\chi_{E}}$ is a projection in the commutant $\mathscr{A}^{\prime}$. Then for any $\alpha \in[0,1]$, we have that

$$
\left(S_{\alpha}+T_{1-\alpha}\right) M_{\chi_{E}}=M_{\chi_{E}}\left(S_{\alpha}+T_{1-\alpha}\right) \text {. }
$$

Note that $\left(S_{\alpha}+T_{1-\alpha}\right) \chi_{[0,1]}=\chi_{[0,1]}$. Therefore we have

$$
\left(S_{\alpha}+T_{1-\alpha}\right) \chi_{E}=\chi_{E} .
$$

For all nonnegative integers $n$, calculating the Fourier coefficients

$$
\left[\left(S_{\alpha}+T_{1-\alpha}\right) M_{\chi_{k}}\right]^{\frown}(n)
$$


of $\left(S_{\alpha}+T_{1-\alpha}\right) M_{\chi_{E}}$ directly, we have that

$$
\left[\left(S_{\alpha}+T_{1-\alpha}\right) M_{\chi_{E}}\right]^{\wedge}(n)=e^{2 \pi n \alpha i} \widehat{\chi_{E}}(n) .
$$

By the fact that $\left(S_{\alpha}+T_{1-\alpha}\right) \chi_{E}=\chi_{E}$ for all $\alpha \in[0,1]$, we get that $\widehat{\chi_{E}}(n)=0$ for all integers $n \neq 0$. Thus $\chi_{E}$ is a constant function, and hence, $M_{\chi_{E}}$ is either 0 or the identity operator. It follows that the commutant $\mathscr{A}^{\prime}$ of $\mathscr{A}$ is trivial.

Corollary 11. Suppose $\theta \in(0,1)$ is irrational. Then the semigroup $\mathscr{S}_{\theta}$ is irreducible.

Proof. It follows directly from Theorem 10.

Remark. The operators $S_{\alpha}$ and $T_{\alpha}(\alpha \in[0,1])$ are so-called Bishop-type operators. Some nice properties of the Bishop-type operators can be found in [2] and in the references at the end of [2].

It is easy to see that the index of nilpotence of operators in $\mathscr{S}_{\theta}$ is not bounded for any irrational $\theta \in(0,1)$. Hadwin et al. [1, Theorem 6] proved that an algebra of nilpotent operators is simultaneously triangularizable if the index of nilpotence is bounded. Thus, it is natural to ask the following question:

Question. Is it true that any semigroup of positive nilpotent operators is reducible if the index of nilpotence is bounded?

\section{REFERENCES}

1. D. Hadwin, E. Nordgren, M. Radjabalipour, H. Radjavi, and P. Rosenthal, $A$ nil algebra of bounded operators on Hilbert space with semisimple norm closure, Integral Equations Operator Theory 9 (1986), 739-743.

2. G. W. MacDonald, Invariant subspaces for Bishop-type operators, J. Funct. Anal. 91 (1990), 287-311.

3. H. H. Schaefer, Topologische nilpotenz irreduzibler operatoren, Math. Z. 117 (1970), 135140.

4. A. R. Sourour, Pseudo-integral operators, Trans. Amer. Math. Soc. 253 (1979), 339-363.

5. Yong Zhong, Functional positivity and invariant subspaces of semigroups of operators, Houston J. Math. 19 (1993), 239-262.

Department of Mathematics, University of Toronto, Toronto, Ontario, Canada M5S $1 \mathrm{~A} 1$

E-mail address: zhong@math.toronto.edu 